\title{
REFLECTIONS IN TOPOLOGICAL ALGEBRAIC STRUCTURES
}

\author{
JULIO HERNÁNDEZ-ARZUSA AND SALVADOR HERNÁNDEZ
}

\begin{abstract}
Let $\mathcal{C}$ be an epireflective category of Top and let $\mathrm{r}_{\mathcal{C}}$ be the epireflective functor associated with $\mathcal{C}$. If $\mathcal{A}$ denotes a (semi)topological algebraic subcategory of Top, we study when $\mathrm{r}_{\mathfrak{C}}(\mathcal{A})$ is an epireflective subcategory of $\mathcal{A}$. We prove that this is always the case for semi-topological structures and we find some sufficient conditions for topological algebraic structures. We also study when the epireflective functor preserves products, subspaces and other properties. In particular, we solve an open question about the coincidence of epireflections proposed by Echi and Lazaar in [6. Question 1.6] and repeated in [7, Question 1.9]. Finally, we apply our results in different specific topological algebraic structures.
\end{abstract}

\section{INTRODUCTION}

In this paper we deal with some applications of epireflective functors in the investigation of topological algebraic structures. In particular, we are interested in the following question: Let $\mathcal{C}$ be an epireflective subcategory of Top (i.e., productive and hereditary, hence containing the 1-point space as the empty product), and let $r_{\mathfrak{C}}$ be the epireflective functor associated with $\mathcal{C}$. If $\mathcal{A}$ denotes a (semi)topological varietal subcategory of Top (that is, a subcategory that is closed under products, subalgebras and homomorphic images), we study when $\mathrm{r}_{\mathfrak{C}}(\mathcal{A})$ is an epireflective subcategory of $\mathcal{A}$. From a different viewpoint, this question has attracted the interest of many researchers recently (cf. [18, 21, 22, 24, 25, 26, 27, 30]) and, to some extent, our motivation for

Date: November 21, 2018.

The second listed author acknowledges partial support by Universitat Jaume I, grant P11B2015-77; Generalitat Valenciana, grant code: PROMETEO/2014/062; and the Spanish Ministerio de Economía y Competitividad, grant MTM-2016-77143-P.

2010 Mathematics Subject Classification. Primary: 54B30, 18B30, 54D10; Secondary: 54H10, $22 \mathrm{~A} 30$

Key Words and Phrases: Topological algebraic structure, Epireflection, Separation axiom, Mal'tsev spaces . 
this research has been to give a unified approach to this topic. First, we recall some definitions and basic facts.

A full subcategory $\mathcal{A}$ of a category $\mathcal{B}$ is reflective if the canonical embedding of $\mathcal{A}$ in $\mathcal{B}$ has a left adjoint $\mathrm{r}_{\mathcal{A}}: \mathcal{B} \longrightarrow \mathcal{A}$ (called reflection). Thus for each $\mathcal{B}$-object $B$ there exists an $\mathcal{A}$-object $\mathrm{r}_{\mathcal{A}} B$ and and a $\mathcal{B}$-morphism $\mathrm{r}_{(B, \mathcal{A})}: B \rightarrow \mathrm{r}_{\mathcal{A}} B$ such that for each $\mathcal{B}$-morphism $f: B \rightarrow A$ to an $\mathcal{A}$-object $A$, there exists a unique $\mathcal{A}$-morphism $\bar{f}: \mathrm{r}_{\mathcal{A}} B \rightarrow A$ such that the following diagram commutes

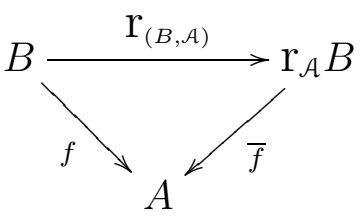

The pair $\left(\mathrm{r}_{\mathcal{A}} B, \mathrm{r}_{(B, \mathcal{A})}\right)$ is called the $\mathcal{A}$-reflection of $B$ and the morphism $\mathrm{r}_{(B, \mathcal{A})}$ is called $\mathcal{A}$-reflection arrow. If all $\mathcal{A}$-reflection arrows are epimorphisms, then the subcategory $\mathcal{A}$ is said to be epireflective. The functor $\mathrm{r}: \mathcal{B} \rightarrow \mathcal{A}$, which is called the reflector, assigns to each $\mathcal{B}$-morphism $f: X \longrightarrow Y$, the $\mathcal{A}$-morphism $\mathrm{r}_{\mathcal{A}}(f)$ that is determined by the following commutative diagram

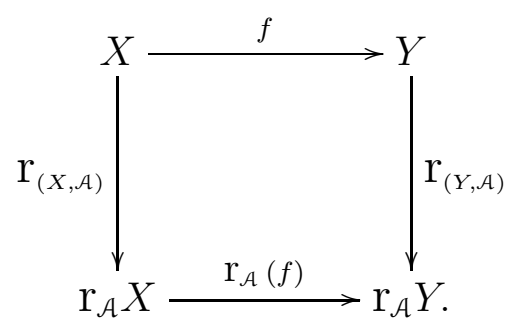




\section{BASIC FACTS}

We collect in this section some known facts about epireflective categories that will be used along the paper. Here, we look at the category Top of topological spaces and continuous functions. Following Kennison [16], by a topological property $\mathcal{P}$, we mean a full subcategory of Top which is closed under the formation of isomorphic ( = homeomorphic) objects. In general, such subcategories are called isomorphism-closed, or replete. When speaking about subcategories, we will always suppose that they are full and replete. A topological property $\mathcal{P}$ is hereditary (resp. divisible, productive, or coproductive) if the objects of $\mathcal{P}$ are closed under the formation of subspaces (resp. quotient spaces, product spaces, or coproduct spaces.) Here, the terms "product", "coproduct", "quotient space" and "subspace" are used in their topological sense. In particular, Kennison proved that a full subcategory $\mathcal{P}$ of Top is epireflective if and only if $\mathcal{P}$ is hereditary and productive (cf. [16]). Well known examples of reflective subcategories in Top are: the classes of all $T_{0}, T_{1}, T_{2}$, and $T_{3}$ spaces, the class of all regular spaces, the completely regular spaces, the class of all totally disconnected spaces (cf. [16]). For $T_{3.5}$ spaces there exist the following reflections: the Stone-Cech compactification, the Hewitt realcompactification.

Let $\mathcal{C}$ denote an epireflective subcategory of Top. That is, for each topological space $X$, there exists an associated topological space $\mathrm{r}_{\mathcal{C}} X \in \mathcal{C}$ and a surjective continuous function $\mathrm{r}_{(X, \mathcal{C})}: X \longrightarrow \mathrm{r}_{\mathfrak{e}} X$ such that for every continuous function $f: X \longrightarrow Y$, with $Y \in \mathcal{C}$, there exists a continuous function $\tilde{f}: \mathrm{r}_{\mathcal{C}} X \longrightarrow Y$ (unique by surjectivity of $\left.\mathrm{r}_{(X, \mathrm{e})}\right)$ such that the following diagram commutes 


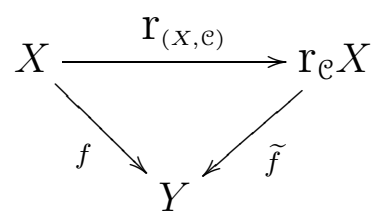

For a topological space $X$ and a continuous map $f: X \longrightarrow Y$ between topological spaces we write $\mathrm{r}_{\mathfrak{C}} X$ and $\mathrm{r}_{\mathfrak{C}}(f): \mathrm{r}_{\mathfrak{C}} X \longrightarrow \mathrm{r}_{\mathfrak{C}} Y$ for the image of $X, Y$ and $f$ by the epireflection functor associated to $\mathcal{C}$. If the subcategory $\mathcal{C}$ is clear from the context, we omit it from the index. Furthermore, the space $\mathrm{r}_{\mathcal{C}} X$ is uniquely determined up to a homeomorphism, and $r_{(X, e)}$ is uniquely determined up to composition from the right with a homeomorphism. It is well known that for every subcategory $\mathcal{A}$ of Top there exists a smallest epi-reflective subcategory $\mathcal{C}(\mathcal{A})$ in Top containing $\mathcal{A}$, namely spaces homeomorphic to subspaces of products of spaces from $\mathcal{A}$, (cf. [16]). It is said that $\mathcal{A}$ generates $\mathcal{C}(\mathcal{A})$. In case there is a single space $X$ with $\mathcal{C}(\{X\})=\mathcal{C}$, we say that $X$ generates $\mathcal{C}$ and $\mathcal{C}$ is called simply generated by $X$. (cf. [12, 13]). For example the class $\mathbf{T o p}_{\mathbf{0}}$ of $T_{0}$ spaces is generated by the Sierpiński space. However, the class Top $_{\mathbf{1}}$ of $T_{1}$ spaces is not simply generated. In fact, the class $\mathbf{T o p}_{\mathbf{1}}$ is generated by the family of cofinite spaces. Furthermore, given an infinite cardinal $\kappa$, the space $\kappa_{\text {cof }}$, which is the set $\kappa$ equipped with the cofinite topology, simply generates the epireflective subcategory of Top generated by all $T_{1}$-spaces of cardinality at most $\kappa$ (see [9, 12]).

For self-completeness, we recall below a realization of the epireflection associated to a epireflective subcategory $\mathcal{C}(\mathcal{A})$ that is generated by a subcategory $\mathcal{A}$ of Top.

Let $\mathbf{F}(X, \mathcal{A})$ denote the class of all continuous functions of $X$ onto spaces in $\mathcal{A}$. We set the following equivalence relation: for $f: X \rightarrow Y$ and $g: X \rightarrow Z$ in $\mathbf{F}(X, \mathcal{A})$, it is said that $f$ and $g$ are equivalent, $f \sim g$, if there is a homeomorphism $\psi: Y \rightarrow Z$ such 
that $g=\psi \circ f$. Set $\kappa=|X|$. Since every continuous image of $X$ can be considered as a subset of $\kappa$, the family of equivalence classes $\mathbf{E}(X, \mathcal{A})=\mathbf{F}(X, \mathcal{A}) / \sim$ defines a set. Let $E=E(X, \mathcal{A})$ be the set defined by selecting a fixed element in each equivalence class in $\mathbf{E}(X, \mathcal{A})$ and let $\varphi_{\mathcal{A}(X)}=\Delta_{E} X: X \rightarrow \prod_{f \in E} f(X)$ be the diagonal map of $X$ into the product $\Pi_{E} X=\prod_{f \in E} f(X)$. We have that $\varphi_{\mathcal{A}(X)}$ is a continuous function from $X$ into $\Pi_{E} X$ and, since $\Pi_{E} X \in \mathcal{E}(\mathcal{A})$, it follows that $\varphi_{\mathcal{A}(X)}(X) \in \mathcal{E}(\mathcal{A})$. It is easy to check that $\left(\mathcal{A}(X), \varphi_{\mathcal{A}(X)}\right)$ satisfies the universal property of a reflection. Indeed, let $h: X \longrightarrow Y$ be a continuous function from $X$ into $Y \in \mathcal{A}$. Then, there exists $f \in \mathbf{F}(X, \mathcal{A})$, say $f: X \rightarrow Z$, such that $f \sim h$. Let $\psi: Z \rightarrow Y$ be a homeomorphism with $h=\psi \circ f$ and let $\pi_{f}$ be the canonical projection of $\Pi_{E} X$ in $f(X)$. We have $f=\pi_{f} \circ \varphi_{\mathcal{A}(X)}$, which yields $h=\psi \circ\left(\pi_{f} \circ \varphi_{\mathcal{A}(X)}\right)=\left(\psi \circ \pi_{f}\right) \circ \varphi_{\mathcal{A}(X)}$. The general case, when $Y \in \mathcal{E}(\mathcal{A})$, follows easily observing that $Y$ is a subspace of a product of spaces in $\mathcal{A}$.

The following facts are easily verified.

Proposition 2.1. Let $\mathcal{C}_{1}$ and $\mathcal{C}_{2}$ be epireflective subcategories in Top such that $\mathcal{C}_{2} \subseteq$ $\mathrm{C}_{1}$. Then the pair

$\left(r_{\mathrm{C}_{2}}\left(r_{\mathrm{C}_{1}} X\right), r_{\left(r_{\mathrm{e}_{1}} X, \mathrm{C}_{2}\right)}\right)$ is a realization of the $\mathcal{C}_{2}$-reflection of $X$ in $\mathbf{T o p}$.

Definition 2.2. A class $\mathcal{C}$ in Top is closed under supertopologies if whenever $(X, \tau) \in \mathcal{C}$ and $\rho$ is a topology on $X$ finer than $\tau$, it follows that $(X, \rho) \in \mathcal{C}$.

The following result, whose proof is folklore, clarifies the action the epi-reflection functor for subcategories closed under supertopologies. (see [13] for the proof, which is straightforward anyway). 
Theorem 2.3. An epireflective subcategory $\mathcal{C}$ in Top is closed under supertopologies if and only if the reflection arrow $r_{(X, \mathrm{e})}$ is a quotient mapping.

A topological space is called functionally Hausdorff, or Urysohn, if distinct points can be separated by a real-valued continuous function, or if any two distinct points have disjoint closed neighbourhoods, respectively.

Corollary 2.4. The reflection arrow $r_{(X, \mathrm{e})}$ is a quotient mapping for each of the following subcategories of Top defined by the separation axioms: $T_{0}, T_{1}, T_{2}$, functionally Hausdorff, and Urysohn.

The next result gives a general realization of the reflection functor for categories whose reflection arrows are quotients. We omit its easy proof here.

Proposition 2.5. Let $X$ be a topological space and let $\mathcal{C}$ denote an epireflective subcategory of Top whose reflection arrows are quotient maps. If $R_{\mathcal{C}}$ is the intersection of all equivalence relations $R \subseteq X^{2}$ on $X$ such that $X / R \in \mathcal{C}$, then $r_{\mathrm{e}} X=X / R_{\mathcal{e}}$.

By Theorem 2.3, the proposition above applies to the epireflections defined by the separation axioms: $T_{0}, T_{1}, T_{2}$, functionally Hausdorff, and Urysohn. In particular, if $\mathcal{C}_{1}$ and $\mathcal{C}_{2}$ denote the subcategories defined by $T_{1}$ and $T_{2}$, we have the following characterization, whose proof is folklore.

Proposition 2.6. Let $X$ be a topological space and let $R$ be an equivalence relation on $X$. The following assertions are fulfilled:

(1) $X / R$ is $T_{1}$ if and only if each equivalence class in $R$ is closed in $X$. 
(2) If the space $X / R$ is Hausdorff then $R$ is closed in $X \times X$. Conversely, if $R$ is a closed subset of $X \times X$ and in addition the quotient map $\varphi: X \rightarrow X / R$ is open, then $X / R$ is Hausdorff.

Corollary 2.7. Let $X$ be a topological space. Then

(1) $r_{\mathfrak{C}_{1}} X=X / R_{\mathfrak{C}_{1}}$ where $R_{\mathfrak{C}_{1}}$ is the intersection of all equivalence relations whose equivalence classes are closed in $X$.

(2) Let $R_{X}$ be the smallest equivalence relation that is closed in $X \times X$. Then $r_{\mathrm{e}_{2}} X$ is canonically homeomorphic to $r_{\mathrm{e}_{2}}\left(X / R_{X}\right)$.

Proof. The verification of (1) is clear.

As for the proof of (2), by Proposition 2.5, we know that there is an equivalence relation $R_{2}$ on $X$ such that $\mathrm{r}_{\mathrm{C}_{2}} X=X / R_{2}$. Furthermore, Proposition [2.6(2) implies that $R_{2}$ must be closed in $X \times X$. Hence $R_{X} \subseteq R_{2}$. Thus we have the following commutative diagram

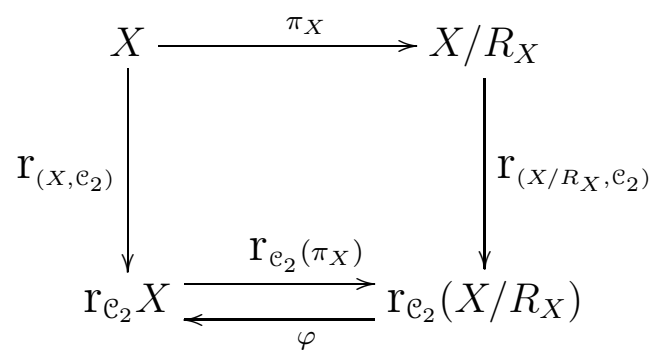

Here, the map $\varphi$ is continuous because $\pi_{X}$ and $\mathrm{r}_{X / R_{X}, \mathrm{e}_{2}}$ are both quotient morphisms. Thus, $\mathrm{r}_{\mathfrak{C}_{2}}\left(\pi_{X}\right)$ and $\varphi$ are each other inverse, which completes the proof. 


\section{EpirefleCtive CATEGORIES IN TOPOLOGICAL AND SEMITOPOLOGICAL}

\section{ALGEBRAIC STRUCTURES}

So far, only categories of topological spaces have been considered. However, our main interest lies on topological algebraic categories. Taking the terminology of Hart and Kunen [11], in what follows an algebraic system $\mathcal{L}$ is a set (possibly empty or infinite) of symbols of constants, symbols of functions (every function symbol has a finiteaa arity $\geq 1$, i.e., $\left.\Phi: X^{n} \rightarrow X\right)$ and a set of equations $\Sigma_{\mathcal{L}}$ that the elements, functions and constants must satisfy. (E.g., $\forall x e x=x$; this contains a constant sign, a binary operation sign and a variable.) An algebraic system is also called a variety, but further we will use the terminology algebraic system.

For a categorical generalization of algebraic, and varietal also called monadic functors, see [1, 14]

A structure $\mathfrak{U}$ for $\mathcal{L}$ is a set $A$ (the domain) together with elements $c_{\mathfrak{U}}$ (of) and functions $\Phi_{\mathfrak{U}}: A^{n} \rightarrow A$, for $n \geq 1$ a natural number, the arity of the respective operation, corresponding to the constants and operations in $\mathcal{L}$ (these we call the specifications of the constants and operations from $\mathcal{L}$ in $\mathfrak{U}$ ), that satisfy the equations established in $\Sigma_{\mathcal{L}}$. E.g., when we talk about groups, it is understood that $\mathcal{L}=\left\{\cdot, i, 1, \Sigma_{\mathcal{L}}\right\}$ (symbols of the product, inverse element, identity and $\Sigma_{\mathcal{L}}$ denotes the equations that define a group). In general, groups (and other algebraic systems) are displayed as $\mathfrak{U}=(A ; \cdot, i, 1)$, avoiding the use of the corresponding set of equations $\Sigma_{\mathcal{L}}$ for short. Here, only algebraic systems that are specified by a set of equations are considered (cf. [10]).

For two structures $\mathfrak{U}$ and $\mathfrak{V}$ for $\mathcal{L}$ we say that $f: \mathfrak{U} \rightarrow \mathfrak{V}$ is algebraically an $\mathcal{L}$ homomorphism if $f\left(c_{\mathfrak{U}}\right)=c_{\mathfrak{B}}$ for each constant symbol $c$ of $\mathcal{L}$, and $f\left(\Phi_{\mathfrak{U}}\left(x_{1}, \ldots, x_{n}\right)\right)=$ $\Phi_{\mathfrak{V}}\left(f\left(x_{1}\right), \ldots, f\left(x_{n}\right)\right)$ for each function symbol $\Phi$ of $\mathcal{L}$, of arity $n \geq 1$. 
A topological structure (resp. semitopological structure) for $\mathcal{L}$ is a pair $(\mathfrak{U}, \tau)$ where $\mathfrak{U}$ is a structure for $\mathcal{L}$, and $\tau$ is a topology on $A$ making all functions in $\mathfrak{U}$ continuous (resp. separately continuous). We write $\mathfrak{U}$ for $(\mathfrak{U}, \tau)$ if the topology is understood.

Let $\mathfrak{U}$ and $\mathfrak{V}$ be two (semi)topological structures of $\mathcal{L}$, and $f: \mathfrak{U} \rightarrow \mathfrak{V}$. The map $f$ is an $\mathcal{L}$-homomorphism from $\mathfrak{U}$ to $\mathfrak{V}$ iff $f$ is continuous and is algebraically an L-homomorphism.

The class consisting of $\mathcal{L}$-topological (resp. $\mathcal{L}$-semitopological) structures and $\mathcal{L}$ morphisms defines a subcategory of Top that will be denoted by $\mathbf{T o p} \mathcal{L}(\operatorname{resp}$. STop $\mathcal{L}$ ). For example, the category of topological groups TopGrp is specified by $\mathcal{L}=(\cdot, i, 1)$ with arities $(2,1,0)$.

We thank the referee for the information and references that follows next.

Remark 3.1. The notions we are dealing with here appeared at the end of the 19-th century in a text by Whitehead [29], but the theory began to develop well only after the theory of lattices was sufficiently well developed, in the thirties of the 20th century. This is the subject of universal algebra. Thus, what we have named an algebraic system, is also called a variety. As a general references to universal algebras, it is pertinent to mention here the volumes [5, 10, 17].

Definition 3.2. Let $\Phi$ be a $n$-ary function on $X$, i.e., $\Phi: X^{n} \rightarrow X$, where $n \geq 1$ is an integer. A $\Phi$-congruence in $X$ is an equivalence relation $R$ in $X$ such that if $x_{i}, y_{i} \in X$, $i=1, \ldots, n$ and $\left(x_{i}, y_{i}\right) \in R$ for $i=1, \ldots n$, then $\left(\Phi\left(x_{1}, \ldots, x_{n}\right), \Phi\left(y_{1}, \ldots, y_{n}\right)\right) \in R$. (Observe that the analogue of this for constants $c$ this is automatically satisfied, since $(c, c) \in R)$. 
Let $\mathcal{L}$ be an algebraic system and let $\mathfrak{U}$ be a structure for $\mathcal{L}$. If $X$ is the domain of $\mathfrak{U}$ and $R$ is an equivalence relation on $X$ that is a $\Phi_{\mathfrak{U} \text {-congruence for all function symbols }}$ $\Phi \in \mathcal{L}$, then we say that $R$ is an $\mathcal{L}$-congruence.

The following proposition are well known, we include them for the reader's sake.

Proposition 3.3. [10, Lem. 2, p. 36] Let $\Phi$ be a n-ary function on $X$ and let $R$ be a $\Phi$-congruence. If $\pi: X \rightarrow X / R$ is the quotient map, then there is an n-ary map $\Phi_{R}:(X / R)^{n} \rightarrow X / R$ defined by $\Phi_{R}\left(\pi\left(x_{1}\right), \ldots, \pi\left(x_{n}\right)\right)=\pi\left(\Phi\left(x_{1}, \ldots, x_{n}\right)\right)$.

Corollary 3.4. [10, Th. 2, p. 58] Let $\mathcal{L}$ be an algebraic system and let $\mathfrak{U}$ be a structure for $\mathcal{L}$. If $X$ is the domain of $\mathfrak{U}$ and $R$ is an $\mathcal{L}$-congruence on $X$, then $X / R$ is the domain for a structure $\mathfrak{V}=\mathfrak{U} / R$ for $\mathcal{L}$, with constant symbols $c / R$ for constant symbols $c \in X$, and with function symbols $\Phi_{\mathfrak{V}}$ satisfying $\Phi_{\mathfrak{V}}\left(\pi\left(x_{1}\right), \ldots, \pi\left(x_{n}\right)\right)=\pi\left(\Phi_{\mathfrak{U}}\left(x_{1}, \ldots, x_{n}\right)\right)$. Here $\pi: X \rightarrow X / R$ is the natural quotient map.

Proposition 3.5. [10, Th. 1, p. 57] Let $\Phi$ and $\Psi$ be n-ary maps on $X$ and $Y$, respectively. If $f: X \rightarrow Y$ is a map such that $f\left(\Phi\left(x_{1}, \ldots, x_{n}\right)\right)=\Psi\left(f\left(x_{1}\right), \ldots, f\left(x_{n}\right)\right)$ for all $\left(x_{1}, \ldots, x_{n}\right) \in X^{n}$, then there is a $\Phi$-congruence $R$ on $X$ and an injective map $\tilde{f}: X / R \rightarrow Y$ that makes the following diagram commutative

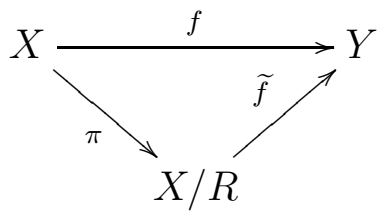

In particular $\tilde{f}\left(\Phi_{R}\left(\pi\left(x_{1}\right), \ldots, \pi\left(x_{n}\right)\right)\right)=\Psi\left(f\left(x_{1}\right), \ldots, f\left(x_{n}\right)\right)$. 
The following result is a generalization of the first isomorphism theorem for arbitrary $\mathcal{L}$-structures.

Theorem 3.6. [10, Th. 1, p. 57] Let $f: X \rightarrow Y$ be an $\mathcal{L}$-homomorphism from $\mathfrak{U}$ to $\mathfrak{V}$. Then there is an $\mathcal{L}$-congruence $R$ on $X$ and an injective map $\tilde{f}: X / R \rightarrow Y$ that makes the following diagram commutative

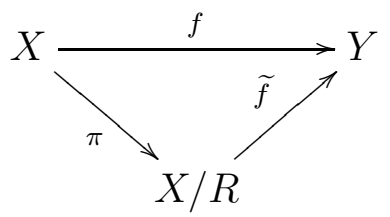

where $\tilde{f}$ is an $\mathcal{L}$-homomorphism from $\mathfrak{U} / R$ to $\mathfrak{V}$.

The next proposition is essential in many subsequent results. Here we present a stronger version than our initial result, for finitely many spaces, which has been kindly offered to us by the referee. Recall that given a family of topological spaces $\left\{X_{i}\right\}_{i \in I}$, a map $f: X_{1} \times \ldots, \times X_{n} \longrightarrow Y$ is named separately continuous when for every $i \in I$ and $\vec{x} \in \prod_{j \neq i} X_{j}$, the map $f_{\vec{x}}: X_{i} \rightarrow Y$, defined by $f_{\vec{x}}(x) \stackrel{\text { def }}{=} f(x ; \vec{x})$, is continuous for every $\vec{x} \in \prod_{j \neq i} X_{j}$, where the symbol ; is used to mean that the variable $\mathrm{x}$ is placed at the coordinate $\mathrm{i}$.

Proposition 3.7. Let $\left\{X_{i}\right\}_{i \in I}$ a family of topological spaces and let $f: \prod_{i \in I} X_{i} \rightarrow Y$ be a separately continuous map into a topological space $Y$. If $\mathrm{C}$ is an epireflective subcategory of Top, then there is a (necessarily unique) separately continuous map $\bar{f}: \prod_{i \in I} r_{\mathrm{e}} X_{i} \rightarrow r_{\mathrm{e}} Y$ 
Proof. Since colimits are preserved by reflections, and actually by all functors having a left adjoint (cf. Adámek-Herrlich-Strecker, Prop. 18.10 (given there in dual formulation), or Herrlich-Strecker, Theorem 27.7), it will suffice to prove that the topology of separate continuity on a product (the so called cross topology) is a final structure, for certain maps from the factors of the product, hence is a colimit of a diagram having objects among the factors of the product.

Indeed, let $X_{i}$, for $i \in I$, be non-empty topological spaces (for some factor the empty space the product of the underlying sets is empty, so there is a unique topology on it.) Here for simplicity later we omit the index set $I$. We define on the underlying set of $\prod X_{i}$ the following topology. Let $\left\langle x_{i}\right\rangle \in \prod X_{i}$ have the following neighbourhood base. We take any open sets $G_{i}$ of $X_{i}$ containing $x_{i}$, and then the neighbourhood base of $\left\langle x_{i}\right\rangle$ will consist of the sets $\prod_{j \neq i}\left\{x_{j}\right\} \times G_{i}$ (a cross). This is the intersection of the topologies $X_{i} \times\left(\prod_{j \neq i} X_{j}\right)_{\text {discr }}$, where $i \in \mathrm{I}$ is arbitrary, and the second factor has the discrete topology. Observe that this second factor can be given also in another way. We take the topological sum of $\left|\prod_{j \neq i} X_{j}\right|$ copies of $X_{i}$. Observe that the underlying set functor $U::$ Top $\rightarrow$ Set has both a left adjoint (discrete topologies on sets) and a right adjoint (indiscrete topologies on sets). Therefore $U$ preserves all limits and colimits from Top to Set, in particular products.

In order to take the intersection of topologies, which is once more a colimit, we have to identify each set $\mathrm{r}_{\mathrm{e}} X_{i} \times \prod_{j \neq i} \mathrm{r}_{\mathrm{e}} X_{j}$ canonically to the set $\prod_{j \in I} \mathrm{r}_{\mathrm{e}} X j$. However, $U$ preserves products, hence this makes the desired identification (by iteration of products).

In particular, the sum diagram, as a colimit, is preserved, therefore we have the sum of $\left|\prod_{j \neq i} X_{j}\right|$ many copies of $\mathrm{r}_{\mathrm{e}} X_{j}$, with the canonical injections from the $\mathrm{r}_{\mathrm{e}} X_{j} \mathrm{~s}$, 
where $j \neq i$. This is once more the product of $\mathrm{r}_{\mathrm{e}} X_{i}$ and a discrete space of cardinality $\left|\prod_{j \neq i} X_{j}\right|$. We ought to reduce this cardinality to $\left|\prod_{j \neq i} \mathrm{r}_{\mathcal{C}} X_{j}\right|$.

Observe that coequalizers, being colimits, are preserved by reflections. However, the image will be only a coequalizer, but not a cointersection of extremal epimorphisms. It will be a cointersection only if the domains of the morphisms (to be coequalized) are the same. This brings in once more that the cardinality $\left|\prod_{j \neq i} X_{j}\right|$ ought to be reduced to $\left|\prod_{j \neq i} \mathrm{r}_{\mathcal{C}} X_{j}\right|$. Now recall that cointersections can be made iteratively. Namely, we divide the maps to be coequalized to (equivalence) classes, take the coequalizer of each class, which is an extremal epimorphism. Now we have a lot of extremal epimorphisms (as many as there are classes) and we have to take the cointersection of them, which is once more an extremal epimorphism. This gives the coequalizer of all considered morphisms.

Now the classes will be formed as follows. One class is formed by all $\left\langle x_{j} \mid j \neq i\right\rangle$, for which the images $\left\langle\mathrm{r}_{\mathcal{C}, X_{j}}\left(x_{j}\right) \mid j \neq i\right\rangle$ are a fixed point of $\prod_{j \neq i} \mathrm{r}_{\mathcal{C}} X_{j}$.

The next corollary follows from Proposition 3.7. However, we include the proof here for the reader's sake.

Corollary 3.8. Let $\left\{X_{1}, \ldots, X_{n}\right\}$ be finitely many topological spaces and let $f: X_{1} \times$ $\cdots \times X_{n} \rightarrow Y$ be a separately continuous map into a topological space $Y$. If $\mathrm{C}$ is an epireflective subcategory of Top, then there is a (necessarily unique) separately continuous map $\bar{f}: r_{\mathfrak{e}} X_{1} \times \ldots r_{\mathcal{C}} X_{n} \rightarrow r_{\mathfrak{C}} Y$ such that $\bar{f}\left(\left(r_{\left(X_{1}, \mathcal{C}\right)}\left(x_{1}\right), \ldots, r_{\left(X_{n}, \mathcal{e}\right)}\left(x_{n}\right)\right)\right)=$ $r_{(Y, \mathcal{C})}\left(f\left(\left(x_{1}, \ldots, x_{n}\right)\right)\right)$.

Proof. In order to simplify the notation, we treat the case $n=2$ only, as this is representative for the general case. The proof for $n>2$ is obtained proceeding by induction. 
For each fixed point $c \in X_{2}$, the map $f_{c}: X_{1} \rightarrow Y$, defined by $f_{c}\left(x_{1}\right):=f\left(x_{1}, c\right)$, is continuous. Accordingly, there exists a continuous function $\mathrm{r}_{\mathfrak{e}}\left(f_{c}\right): \mathrm{r}_{\mathfrak{e}} X_{1} \rightarrow \mathrm{r}_{\mathfrak{e}} Y$ that makes commutative the following digram

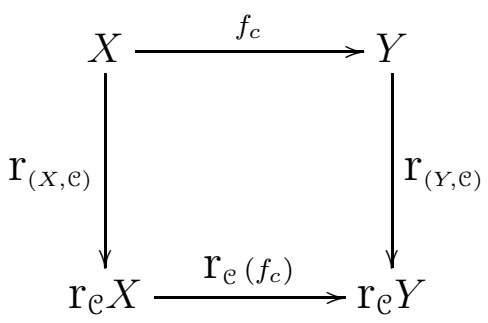

Thus, the map $\mathrm{r}_{\mathcal{C}}\left(f_{c}\right)$ is defined, for each $\mathrm{r}_{\mathfrak{C}} X_{1} \ni x_{1}^{\prime}=\mathrm{r}_{\left(X_{1}, \mathcal{C}\right)}\left(x_{1}\right)$, by

$$
\mathrm{r}_{\mathcal{C}}\left(f_{c}\right)\left(x_{1}^{\prime}\right)=\mathrm{r}_{\mathcal{C}}\left(f_{c}\right)\left(\mathrm{r}_{\left(X_{1}, \mathcal{C}\right)}\left(x_{1}\right)\right)=\mathrm{r}_{(Y, \mathcal{C})}\left(f_{c}\left(x_{1}\right)\right)=\mathrm{r}_{(Y, \mathcal{C})}\left(f\left(x_{1}, c\right)\right)
$$

Furthermore, we have that if $\mathrm{r}_{(X, \mathcal{C})}\left(x_{1}\right)=\mathrm{r}_{(X, \mathcal{C})}\left(u_{1}\right)$ then $\mathrm{r}_{\mathfrak{C}}(f)\left(x_{1}, c\right)=\mathrm{r}_{\mathfrak{C}}(f)\left(u_{1}, c\right)$. This crucial fact will be widely applied in what follows.

The equality above implies that the map $\tilde{f}: \mathrm{r}_{\mathrm{e}} X_{1} \times X_{2} \rightarrow Y$, defined by

$$
\tilde{f}\left(\left(\mathrm{r}_{\left(X_{1}, \mathrm{e}\right)}\left(x_{1}\right), x_{2}\right)\right):=\mathrm{r}_{(Y, \mathrm{e})}\left(f\left(x_{1}, x_{2}\right)\right)
$$

is well defined and separately continuous. Repeating the argument for $\tilde{f}^{d}: X_{2} \rightarrow Y$, defined by $\tilde{f}^{d}\left(x_{2}\right):=\tilde{f}\left(d, x_{2}\right)$ with $d \in \mathrm{r}_{\mathfrak{C}} X_{1}$, for each $d \in \mathrm{r}_{\mathfrak{C}} X_{1}$ there exists a continuous map $\mathrm{r}_{\mathfrak{C}}\left(\tilde{f}^{d}\right): \mathrm{r}_{\mathfrak{e}} X_{2} \rightarrow \mathrm{r}_{\mathfrak{e}} Y$, satisfying

$$
\mathrm{r}_{\mathcal{C}}\left(\tilde{f}^{d}\right)\left(\mathrm{r}_{\left(X_{2}, \mathcal{C}\right)}\left(x_{2}\right)\right)=\mathrm{r}_{(Y, \mathcal{C})}\left(\tilde{f}^{d}\left(x_{2}\right)\right)
$$

It is now clear that the map $\bar{f}: \mathrm{r}_{\mathcal{C}} X_{1} \times \mathrm{r}_{\mathfrak{C}} X_{2} \longrightarrow \mathrm{r}_{\mathfrak{C}} Y$ defined by

$$
\bar{f}\left(\mathrm{r}_{\left(X_{1}, \mathrm{e}\right)}\left(x_{1}\right), \mathrm{r}_{\left(X_{2}, \mathrm{e}\right)}\left(x_{2}\right)\right)=\mathrm{r}_{(Y, \mathrm{e})}\left(f\left(\left(x_{1}, x_{2}\right)\right)\right)
$$

is well defined and separately continuous. 
As a consequence of the previous result, it follows that epireflections respect semitopological structures in the best possible fashion.

Proposition 3.9. Let $\mathbf{S T o p} \mathcal{L}$ be a category of semitopological structures. If $\mathcal{C}$ is an epireflective subcategory of $\mathbf{T o p}$, then $r_{\mathfrak{C}}(\mathbf{S T o p} \mathcal{L}) \subseteq \mathbf{S T o p} \mathcal{L}$. That is, for each $\mathcal{L}$ structure $(\mathfrak{U}, X) \in \mathbf{S T o p} \mathcal{L}$, where $X$ is the domain of $\mathfrak{U}$, we have that $r_{\mathrm{e}} X$ is the domain of an $\mathcal{L}$-structure $\mathfrak{V}$ such that $\left(\mathfrak{V}, r_{\mathfrak{e}} X\right) \in \mathbf{S T o p} \mathcal{L}$. Furthermore, the reflection arrow $r_{(X, \mathcal{e})}: X \rightarrow r_{\mathcal{C}} X$ is an $\mathcal{L}$-homomorphism in $\mathbf{S T o p} \mathcal{L}$ for each $(\mathfrak{U}, X) \in \mathbf{S T o p} \mathcal{L}$.

Proof. Let $(\mathfrak{U}, X)$ be a semitopological structure for $\mathbf{S T o p} \mathcal{L}$. We equip $\mathrm{r}_{\mathfrak{e}} X$ with the algebraic structure $\mathfrak{V}$ built by taking the constants $c_{\mathfrak{V}}:=\mathrm{r}_{(X, \mathcal{C})}\left(c_{\mathfrak{U}}\right)$ for all constants $c \in \mathcal{L}$ and, if $\Phi \in \mathcal{L}$ is a separately continuous $n$-ary function symbol, then we apply Corollary 3.8 in order to define, for $x_{i}^{\prime} \in \mathrm{r}_{\mathcal{C}} X$, with $x_{i}^{\prime}=\mathrm{r}_{(X, \mathcal{C})}\left(x_{i}\right)$, for $x_{i} \in X$, as

$$
\Phi_{\mathfrak{V}}\left(\left(\mathrm{r}_{(X, \mathrm{C})}\left(x_{1}\right), \ldots, \mathrm{r}_{(X, \mathcal{C})}\left(x_{n}\right)\right)\right)=\mathrm{r}_{(X, \mathcal{C})}\left(\Phi_{\mathfrak{U}}\left(\left(x_{1}, \ldots, x_{n}\right)\right)\right)
$$

This definition implies that $\Phi_{\mathfrak{V}}:\left(\mathrm{r}_{\mathcal{C}} X\right)^{n} \rightarrow \mathrm{r}_{\mathfrak{C}} X$ is a well defined separately continuous $n$-ary function symbol for all $\Phi \in \mathcal{L}$. Thus $\mathrm{r}_{\mathcal{C}} X$ is equipped with a semitopological $\mathcal{L}$-structure canonically inherited from the $\mathcal{L}$-structure in $X$. Furthermore, it is also clear that $\mathrm{r}_{(X, \mathcal{e})}$ is a continuous $\mathcal{L}$-homomorphism.

We are now in position of establishing the main result in this section.

Theorem 3.10. Let $\mathbf{S T o p} \mathcal{L}$ be a category of semitopological structures. If $\mathcal{C}$ is an epireflective subcategory of Top, then $r_{\mathfrak{C}}(\mathbf{S T o p} \mathcal{L})$ is an epireflective subcategory of $\mathrm{STop} \mathcal{L}$.

Proof. First, observe that, since $\mathrm{r}_{(X, \mathcal{C})}: X \rightarrow \mathrm{r}_{\mathcal{e}} X$ is an $\mathcal{L}$-homomorphism for all $\mathcal{L}$ structure $(\mathfrak{U}, X) \in \mathbf{S T o p} \mathcal{L}$, it follows that the equivalence relation $\left\{\left(x_{1}, x_{2}\right) \in X^{2}\right.$ : 
$\mathrm{r}_{(X, \mathrm{e})}\left(x_{1}\right)=\mathrm{r}_{(X, \mathrm{e})}\left(x_{2}\right)$ is an $\mathcal{L}$-congruence in $X$ for all $(\mathfrak{U}, X) \in \mathbf{S T o p} \mathcal{L}$. By Proposition 3.9, we know that $\mathrm{r}_{\mathcal{C}}(\mathbf{S T o p} \mathcal{L})$ is equipped with a semicontinuous $\mathcal{L}$-structure, where the reflection arrows $\mathrm{r}_{(X, \mathrm{e})}$ are epimorphisms in Top. Thus it will suffice to show that $\mathrm{r}_{\mathrm{e}}$ preserves $\mathcal{L}$-morphisms.

Let $(\mathfrak{U}, X)$ and $\left(\mathfrak{U}^{\prime}, Y\right)$ be two semitopological structures in $\mathbf{S T o p} \mathcal{L}$ and let $\left(\mathfrak{V}, \mathrm{r}_{\mathfrak{C}} X\right)$ and $\left(\mathfrak{V}^{\prime}, \mathrm{r}_{\mathfrak{e}} Y\right)$ the structures in $\mathbf{S T o p} \mathcal{L}$, with domains $\mathrm{r}_{\mathfrak{e}} X$ and $\mathrm{r}_{\mathfrak{e}} Y$ canonically associated to the former by Proposition 3.9.

Let $f: X \rightarrow Y$ be a continuous $\mathcal{L}$-morphism. If $\Phi \in \mathcal{L}$ is a separately continuous $n$-ary function symbol, by the commutativity of the diagram

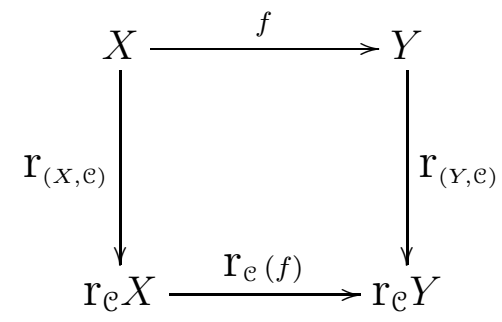

it follows

(1)

$$
\begin{aligned}
\mathrm{r}_{\mathcal{C}}(f)\left(\Phi_{\mathfrak{V}}\left(\mathrm{r}_{(X, \mathcal{C})}\left(x_{1}\right) \cdots, \mathrm{r}_{(X, \mathcal{C})}\left(x_{n}\right)\right)\right) & =\mathrm{r}_{\mathcal{C}}(f)\left(\mathrm{r}_{(X, \mathcal{C})}\left(\Phi_{\mathfrak{U}}\left(\left(x_{1}, \cdots, x_{n}\right)\right)\right)\right) \\
& =\mathrm{r}_{(Y, \mathcal{C})}\left(f\left(\Phi_{\mathfrak{U}}\left(\left(x_{1}, \cdots, x_{n}\right)\right)\right)\right) \\
& =\mathrm{r}_{(Y, \mathcal{C})}\left(\Phi_{\mathfrak{U}^{\prime}}\left(\left(f\left(x_{1}\right) \cdots, f\left(x_{n}\right)\right)\right)\right) \\
& =\Phi_{\mathfrak{V}^{\prime}}\left(\left(\mathrm{r}_{(Y, \mathcal{C})}\left(f\left(x_{1}\right)\right), \cdots, \mathrm{r}_{(Y, \mathcal{C})}\left(f\left(x_{n}\right)\right)\right)\right. \\
& =\Phi_{\mathfrak{V}^{\prime}}\left(\left(\mathrm{r}_{\mathcal{C}}(f)\left(\mathrm{r}_{(X, \mathcal{C})}\left(x_{1}\right)\right) \cdots, \mathrm{r}_{\mathcal{C}}(f)\left(\mathrm{r}_{(X, \mathcal{C})}\left(x_{n}\right)\right)\right)\right) .
\end{aligned}
$$


Theorem 3.10 allows us to obtain a neat realization of epireflections whose reflection arrows are quotient maps. Remark that we have shown that the epireflection functor in Top coincides with the epireflection functor in $\mathbf{S T o p} \mathcal{L}$.

Corollary 3.11. Let $\mathbf{S T o p} \mathcal{L}$ be a category of semitopological structures. If $\mathcal{C}$ is an epireflective subcategory of Top whose reflection arrows $r_{(X, \mathrm{e})}$ are quotient maps then $r_{\mathrm{e}} X=X / R_{\mathcal{e}}$ for all $(\mathfrak{U}, X) \in \mathbf{S T o p} \mathcal{L}$, where $R_{\mathcal{C}}$ coincides with the intersection of all $\mathcal{L}$-congruences $R$ such that $X / R \in \mathcal{C}$.

Proof. It suffices to apply Proposition 2.5 and Theorem 3.10.

On the other hand, using Corollary 2.7, we obtain

Corollary 3.12. Let $\mathbf{S T o p} \mathcal{L}$ be a category of semitopological structures and let $X$ be a space in $\mathbf{S T o p} \mathcal{L}$. Then $r_{\mathrm{e}_{1}} X=X / R_{\mathrm{C}_{1}}$ where $R_{\mathrm{C}_{1}}$ is the intersection of all equivalence relations such that equivalence classes are closed in $X$, furthermore $R_{\mathfrak{C}_{1}}$ is an L-congruence in $X$.

The following result was established by Tkachenko [26, Theorem 3.4] for semitopological groups. Here, we obtain a variant of his proof as an application of our results.

Corollary 3.13. Let $\mathcal{L}$ denote the algebraic system defined by groups and let $\mathbf{S T o p} \mathcal{L}$ be the corresponding category semitopological groups (group operation is separately continuous). Then $r_{\mathrm{C}_{1}} G=G / H$ for all $G \in \mathbf{S T o p} \mathcal{L}$, where $H$ is the intersection of all closed subgroups of $G$.

Proof. First, remark that if $R$ is an $\mathcal{L}$-congruence defined on $G$, then the $R$-equivalence classes are cosets of the normal subgroup $H_{R} \stackrel{\text { def }}{=}\left\{x \in G:\left(x, e_{G}\right) \in R\right\}$. Thus, using 
Corollary 3.12, we obtain that $\mathrm{r}_{\mathrm{C}_{1}} G=G / H$, where $H$ is a normal closed subgroup of $G$. Let us see that $H$ the smallest closed subgroup of $G$. Indeed if $K$ is a closed subgroup of $G$, then $G / K$ is a $T_{1}$ space, therefore if $\pi: G \rightarrow G / K$ is the respective quotient mapping, we have a group homomorphism $\mathrm{r}_{\mathfrak{C}_{1}}(\pi): G / H \rightarrow G / K$ that makes the following diagram

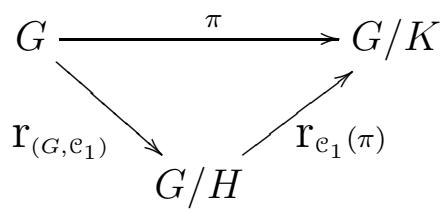

commutative. This obviously means that $H \subseteq K$.

\section{Products}

In this section we deal with the epireflections that preserve products, which is a crucial fact in order to study the preservation of topological structures.

Let $\mathcal{C}$ be an epireflective category in Top and let $\left\{X_{i}\right\}$ a set (resp. finite set) of topological spaces. Then we have the following commutative diagram

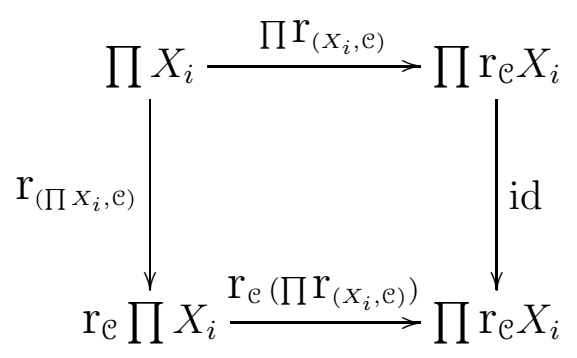

Set $\mu_{\mathcal{e}} \stackrel{\text { def }}{=} \mathrm{r}_{\mathcal{C}}\left(\prod \mathrm{r}_{\left(X_{i}, \mathcal{e}\right)}\right)$. We notice that $\mu_{\mathcal{e}}$ is defined uniquely by the condition that the following diagram commutes for every $j$ 


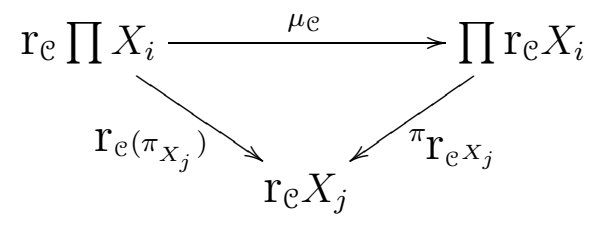

here $\pi_{\mathrm{r}_{\mathrm{e} x_{j}}}$ denotes the respective canonical projection. If $\mu_{\mathrm{e}}$ is a homeomorphism onto $\prod r_{e} X_{i}$ for every family of topological spaces (resp. finite family of topological spaces) then we say that $\mathrm{e}$ preserves products (resp. e preserves finite products).

For example, from [20, Prop. 6.2.1], there follows the following sufficient condition for the preservation of finite products.

Proposition 4.1. Let $\mathcal{C}$ be an epireflective category in Top and let $\left\{X_{i}: i \in I\right\}$ be a set of topological spaces such that $r_{(X, \mathrm{e})}$ is open for all $X \in \mathbf{T o p}$. Then $\mathrm{C}$ preserves the product $\prod_{i \in I} X_{i}$

Proof. Clearly the map $\mu_{\mathfrak{e}}$ defined above is onto. To prove that $\mu_{\mathfrak{e}}$ is injective, it will suffice to repeat mutatis mutandis the argument used by T. Ishii in [20, Prop. 6.2.1]. Therefore, we have shown that $\mu_{\mathrm{e}}$ is a bijection. Moreover, since every reflection arrow $\mathrm{r}_{(X, \mathrm{e})}$ is open, it follows that the reflection arrow $\prod_{i \in I} \mathrm{r}_{\left(X_{i}, \mathrm{e}\right)}$ is also open. Therefore, we have the commutative diagram

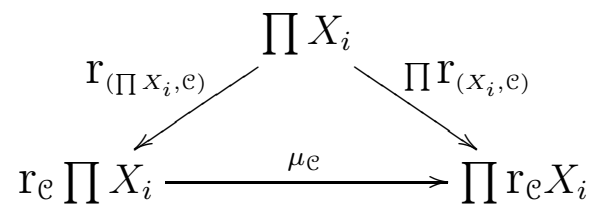

Since both $\mathrm{r}_{\left(\prod X_{i}, \mathrm{e}\right)}$ and $\prod \mathrm{r}_{\left(X_{i}, \mathrm{e}\right)}$ are open and $\mu_{\mathrm{e}}$ is continuous, it follows that $\mu_{\mathrm{e}}$ is a homeomorphism. 
It is a well-known fact that the $T_{0}$-reflection in Top preserves all products but the $T_{1}, T_{2}$ and $T_{3}$ reflections do not preserve all finite products (cf. [15, §1]).

The following result improves Proposition 3.8 for epireflections that preserve finite products. Again, we thank the referee for letting us notice that our results can be extended to infinite products.

Proposition 4.2. Let $\left\{X_{i}: i \in I\right\}$ be a set of topological spaces and let $f: \prod_{i \in I} X_{i} \longrightarrow Y$ be a continuous map into a topological space $Y$. If $\mathcal{C}$ is an epireflective subcategory of Top such that the epireflection preserves the product $\prod_{i \in I} X_{i}$, then there is a (necessarily unique) continuous map $\bar{f}: \prod_{i \in I} r_{\mathrm{e}} X_{i} \longrightarrow r_{\mathrm{e}} Y$ such that

$$
\bar{f}\left(\left\langle r_{\left(X_{i}, \mathcal{C}\right)}\left(x_{i}\right)\right\rangle\right)=r_{(Y, \mathcal{C})}\left(f\left(\left\langle x_{i}\right\rangle\right)\right)
$$

Proof. By hypothesis, the canonical homeomorphism

$$
\mu_{\mathfrak{C}}: \mathrm{r}_{\mathcal{C}} \prod_{i \in I} X_{i} \rightarrow \prod_{i \in I} \mathrm{r}_{\mathfrak{e}} X_{i}
$$

satisfies that the inverse mapping defined by $\mu_{\mathrm{e}}^{-1}\left(\left\langle\mathrm{r}_{\left(X_{i}, \mathrm{e}\right)}\left(x_{i}\right)\right\rangle\right)=\mathrm{r}_{\left(\prod_{i \in I} X_{i}, \mathrm{e}\right)}\left(\left\langle x_{i}\right\rangle\right)$ is well defined (i.e., it depends only on $\mathrm{r}_{\left(X_{i}, \mathrm{e}\right)}\left(x_{i}\right)$ and not on the choice of $x_{i}$ ), and is continuous. Therefore, we have the following commutative diagram:

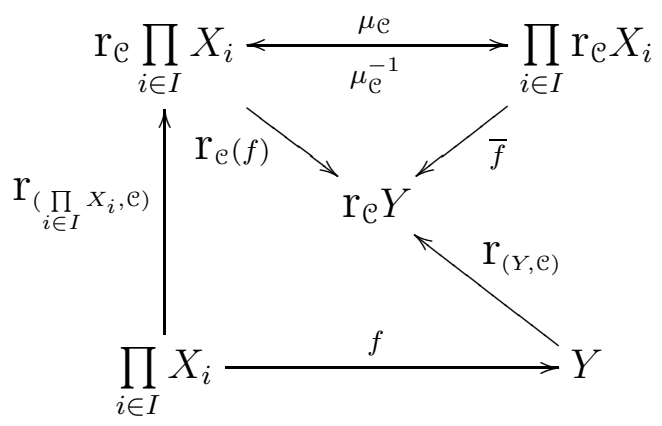


Hence $\bar{f}=\mathrm{r}_{\mathfrak{C}}(f) \circ \mu_{\mathfrak{e}}^{-1}$ is a continuous map. The equality stated in Prop. 4.2 follows from the commutativity of the lower square in the diagram.

The following result improves Theorem 3.10 when the reflection functor preserves products.

Theorem 4.3. Let $\operatorname{Top} \mathcal{L}$ be a category of topological structures. If $\mathcal{C}$ is an epireflective subcategory of Top such that the epireflection preserves products, then $r_{\mathcal{C}}(\mathbf{T o p} \mathcal{L})$ is an epireflective subcategory of $\operatorname{Top} \mathcal{L}$.

Proof. It suffices to observe that $\Phi_{\mathrm{r}_{\mathrm{e}} X}$ is continuous for every $n$-ary function symbol $\Phi \in \mathcal{L}$ as a consequence of Proposition 4.2 .

Corollary 4.4. Let $\operatorname{Top} \mathcal{L}$ be a category of topological structures and let $\mathrm{C}_{0}$ be the class of $T_{0}$, then $r_{\mathrm{C}_{0}}(\operatorname{Top} \mathcal{L})$ is an epireflective subcategory of $\operatorname{Top} \mathcal{L}$.

Proof. The classes of $T_{0}$ spaces is both epireflective and preserve products in Top.

Remark 4.5. Let TopGrp denote the category of topological groups and let $\mathcal{C}_{0}$ denote the epireflective subcategory of $T_{0}$ spaces in Top. Then $r_{\mathfrak{C}_{0}}$ (TopGrp) is an epireflective subcategory of TopGrp. Furthermore, since every $T_{0}$ topological group is $T_{3.5}$, it follows that every member in $\mathrm{r}_{\mathfrak{C}_{0}}$ (TopGrp) is $T_{3.5}$. In other words, for topological groups, the $T_{0}$ reflection and the $T_{3.5}$ reflection coincide.

There are more general structures than groups where the epireflective subcategories that are closed under finer topologies preserve products. An example of this are Mal'tsev spaces. 
Definition 4.6. A Mal'tsev operation on a topological space $X$ is a map $\Phi: X^{3} \rightarrow X$ satisfying the identity $\Phi(x, x, y)=\Phi(y, x, x)=y$ for all $x, y \in X$. A space is a topological Mal'tsev space if it admits a continuous Mal'tsev operation.

For example, if $G$ is a topological group, then the map $(x, y, z) \mapsto x y^{-1} z$ is a Mal'tsev operation on $G$. Hence every topological group is a Mal'tsev space. In like manner, a semitopological Mal'tsev space is a space that admits a separately continuous Mal'tsev operation.

The classes TopMlt (resp. STopMlt) of topological (resp. semitopological) Mal'tsev spaces are algebraic systems in Top with the continuous maps that respect these algebraic structures as arrows.

According to Gartside, Reznichenko and Sipacheva 8] topological Mal'tsev spaces were introduced by Uspenskij in [28] and have subsequently been studied by several authors. In this section we deal with these spaces and our main motivation is to transfer much of the behavior of topological groups to Mal'tsev spaces. We will see that most epireflective functors that preserve the topological group structure also respect the topological Mal'tsev operation.

The following result is attributed to Mal'tsev [19] by Reznichenko and Uspenskij [23, 4.11]. Our formulation is somewhat more general.

Lemma 4.7. Let $(X, \Phi)$ be a semitopological Mal'tsev space and let $R$ be a $\Phi$-congruence in $X$. Then the quotient map $\pi: X \rightarrow X / R$ is open.

Proposition 4.8. Let $(X, \Phi)$ be a a semitopological Mal'tsev space and let $\mathrm{C}$ be an epireflective class in Top closed under supertopologies. Then the reflection arrow $r_{(X, \mathrm{e})}$ is an open map. 
Proof. By Theorem [3.10, we know that $\left(\mathrm{r}_{\mathfrak{C}} X, \mathrm{r}_{\mathfrak{C}}(\Phi)\right)$ is a semitopological Mal'tsev space. Furthermore, the reflection arrow $\mathrm{r}_{(X, \mathrm{e})}$ is a $\Phi$-homomorphism. By Theorem 3.6. there is a $\Phi$-congruence $R$ such that the following diagram commutes

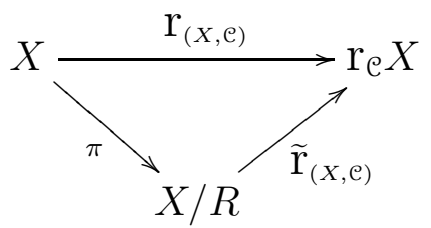

By Theorem 2.3 , is a quotient $\Phi$-homomorphism. Since $\pi$ is open by Lemma 4.7 and $\widetilde{\mathrm{r}}_{(X, \mathrm{e})}$ is one-to-one, it follows that $\mathrm{r}_{(X, \mathcal{e})}$ is open.

From Proposition 4.1 and Proposition 4.8, we obtain.

Theorem 4.9. Let $\mathcal{C}$ be an epireflective category in Top that is closed under finer topologies. If $\left\{X_{i}\right\}$ is a family of semitopological Mal'tsev spaces, then $r_{\mathcal{C}}$ preserves the product of $\left\{X_{i}\right\}$.

Corollary 4.10. If $\mathrm{C}$ denotes the epireflective category of Top defined by any of the following separation axioms: $T_{0}, T_{1}, T_{2}$ and functionally Hausdorff, then $r_{\mathrm{C}}$ preserves arbitrary products of semitopological Mal'tsev spaces.

The next result is an application of the techniques developed in this paper. It shows that the modification of a Mal'tsev space by most separation axioms is a Mal'tsev space.

Theorem 4.11. If $\mathrm{C}$ denotes the epireflective category of Top defined by any of the following separation axioms: $T_{0}, T_{1}, T_{2}$ and functionally Hausdorff, and TopMlt is 
the subcategory of topological Mal'tsev spaces. Then $r_{\mathrm{e}}$ (TopMlt) is an epireflective subcategory of TopMlt.

Proof. Use Corollary 4.10 and Theorem 4.3.

\section{Subspaces}

We say that an epireflection preserves subspaces if the following holds. For $i: A \hookrightarrow X$ an inclusion of a subspace we have that $\mathrm{r}_{\mathfrak{C}}(i): \mathrm{r}_{\mathfrak{C}} A \rightarrow \mathrm{r}_{\mathfrak{C}} X$ is an inclusion of a subspace as well. More exactly: there is an inclusion of a subspace $i: B \hookrightarrow \mathrm{r}_{\mathfrak{e}} X$ and and homeomorphism $h$ such that $j=\mathrm{r}_{\mathfrak{C}}(i) \circ h$. In this section, we study when epireflection functors preserve subspaces in topological algebraic structures.

Definition 5.1. Let $X$ be a topological space. Two subsets $A$ and $B$ of $X$ are said to be completely separated in $X$ if there exists a continuous real valued map $f$ such that $f(a)=0$ for all $a \in A$ and $f(b)=1$ for all $b \in B$.

The space $X$ is said to be functionally Hausdorff if any two different points can be completely separated by a real valued continuous function.

The space $X$ is said to be completely regular if every closed set $F$ of $X$ is completely separated from any point $x \notin F$. A completely regular $T_{1}$-space is called a $T_{3,5}$-space.

A topological space $X$ is said to be Urysohn if any two distinct points have disjoint closed neighhbourhoods.

It is easy to show that epireflection functors do not preserve subspaces in general. In order to see this, consider the epireflective subcategories defined by the separation axioms $T_{0}, T_{1}, T_{2}$, Urysohn, $T_{3}$, functionally Hausdorff, regular, completely regular and $T_{3.5}$, that we denote by $\mathcal{C}_{0}, \mathcal{C}_{1}, \mathcal{C}_{2}, \mathcal{C}_{u}, \mathcal{C}_{3}, \mathcal{C}_{f h}, \mathcal{C}_{r}, \mathcal{C}_{c r}$ and $\mathcal{C}_{3.5}$ respectively. 
Take a set $X$ of arbitrary infinite cardinality and an ideal point $p \notin X$ and set $X^{*} \stackrel{\text { def }}{=} X \cup\{p\}$ and consider the following two topologies on $X^{*}$

$$
\begin{gathered}
\tau_{1}=\left\{U \subseteq X^{*}: p \notin U\right\} \cup\left\{X^{*}\right\} \\
\tau_{2}=\left\{U \subseteq X^{*}: p \in U\right\} \cup\{\emptyset\} .
\end{gathered}
$$

Set $X_{i} \stackrel{\text { def }}{=}\left(X^{*}, \tau_{i}\right), 1 \leq i \leq 2$ and pick a point $x$ in $X_{1}$. Since every neighbourhood of $p$ in $X_{1}$ contains $x$, we have $\mathrm{r}_{\left(X_{1}, \mathrm{e}_{i}\right)}(x)=\mathrm{r}_{\left(X_{1}, \mathrm{e}_{i}\right)}(p)$ for each separation axiom in $\left\{T_{1}, T_{2}\right.$, Urysohn, functionally Hausdorff, regular, completely regular and $\left.T_{3.5}\right\}$. That is to say $\mathrm{r}_{\left(X_{1}, \mathrm{e}_{i}\right)}$ is a single-valued map. Now, take $X$, which is a discrete, dense, open subset of $X_{1}$. We have that $\mathrm{r}_{\mathfrak{C}_{i}} X=X \neq \mathrm{r}_{\left(X_{1}, \mathcal{C}_{i}\right)}(X)=\mathrm{r}_{\left(X_{1}, \mathfrak{e}_{i}\right)}(p)$.

As for the $\mathcal{C}_{r}\left(\right.$ resp. $\left.\mathcal{C}_{c r}\right)$ reflection, remark that no nonempty closed subset of $X_{1}$ is contained in a proper open subset. Therefore $\mathrm{r}_{\mathcal{C}_{r}} X_{1}=\mathrm{r}_{\mathcal{C}_{c r}} X_{1}$ is the indiscrete space and again $\mathrm{r}_{\mathfrak{e}_{r}} X=\mathrm{r}_{\mathfrak{e}_{c r}} X=X$, which yields $\mathrm{r}_{\left(X_{1}, \mathrm{e}_{r}\right)}(X) \neq \mathrm{r}_{\mathfrak{e}_{r}}$ and $\mathrm{r}_{\left(X_{1}, \mathfrak{e}_{c r}\right)}(X) \neq \mathrm{r}_{\mathfrak{C}_{c r}}$. This completes the proof for open subsets. For closed subsets, it suffices to take the space $X_{2}$.

Definition 5.2. Let $X$ be a topological space and let $\mathcal{A}$ be a subset of Top. A subset $A$ of $X$ is said $\mathcal{A}$-oset if there is a space $Y \in \mathcal{A}$ and a continuous map $f: X \longrightarrow Y$ such that $A=f^{-1}(U)$ for some open subset $U$ of $Y$. It is clear that the family of all $\mathcal{A}$-osets forms a subbase in $X$ for the initial topology $\tau_{\mathcal{A}}$, with respect to all continuous maps $X \rightarrow Y \in \mathcal{A}$. The subsets $G \in \tau_{\mathcal{A}}$ are called $\mathcal{A}$-open. A subset $F$ of $X$ is said $\mathcal{A}$-closed, if $X \backslash F$ is $\mathcal{A}$-open.

In case $\mathcal{A}=\mathcal{C}_{i}$ for some $i \in\left\{T_{1}, T_{2}, u, r, f h, c r, T_{3.5}\right\}$, we will use the symbolism $\mathbf{T}_{i}$-open for short. 
Lemma 5.3. Let $X$ be a topological space and let $\mathcal{C}=\mathcal{C}(\mathcal{A})$ be an epireflective subcategory of Top that is generated by a family of spaces $\mathcal{A} \subseteq$ Top. Given a subset $A$ of $X$, the following assertions are equivalent:

(a) A is $\mathrm{C}$-open (res. C-closed).

(b) $A=r_{(X, \mathcal{C})}^{-1}(U)$, for some open (resp. closed) subset $U$ of $r_{\mathrm{e}} X$.

(c) $A$ is $\mathcal{A}$-open (res. $\mathcal{A}$-closed)

Proof. $(a) \Rightarrow(b)$. Let $A$ be a $\mathcal{C}$-oset subset of $X$. Then there are $Y \in \mathcal{C}, V$ open in $Y$ and a continuous map $f: X \longrightarrow Y$ such that $f^{-1}(V)=A$. By the functorial definition of epireflections, there exists a continuous map $g: \mathrm{r}_{\mathfrak{C}} X \longrightarrow Y$ such that $g \circ \mathrm{r}_{(X, \mathcal{e})}=f$. Therefore $A=f^{-1}(V)=\mathrm{r}_{(X, \mathrm{e})}^{-1}\left(g^{-1}(V)\right)$ and it suffices to take $U=g^{-1}(V)$. Now if $A \subseteq X$ is $\mathcal{C}$-open, then $A=\bigcup_{i \in I}\left(\bigcap_{j \in J_{i}} A_{i j}\right)$, where each $J_{i}$ is finite and $A_{i j}=\mathrm{r}_{(X, \mathcal{C})}^{-1}\left(U_{i j}\right)$ for some open subset $U_{i j}$ of $\mathrm{r}_{\mathcal{C}} X$. Then

$$
A=\bigcup_{i \in I}\left(\bigcap_{j \in J_{i}} \mathrm{r}_{(X, \mathrm{e})}^{-1}\left(U_{i j}\right)\right)=\mathrm{r}_{(X, \mathcal{C})}^{-1}\left(\bigcup_{i \in I}\left(\bigcap_{j \in J_{i}} U_{i j}\right)\right)
$$

and we may choose

$$
U \stackrel{\text { def }}{=} \bigcup_{i \in I}\left(\bigcap_{j \in J_{i}} U_{i j}\right)
$$

$(b) \Rightarrow(c)$. We have seen in Section 2 that the space $\mathrm{r}_{\mathrm{e}} X$ can be realized as the diagonal of a product $\Pi_{E} X=\prod_{f \in E} Y_{f}$ where $Y_{f} \in \mathcal{A}$ for all $f \in E$ and $f$ stands for a surjective continuous map $f: X \rightarrow Y_{f}$. Thus, the family $\left\{\pi_{f}^{-1}(W) \cap \mathrm{r}_{\mathrm{e}} X\right.$ : $W$ open in $\left.Y_{f}, f \in E\right\}$ form an open subbase in $\mathrm{r}_{\mathfrak{e}} X$ and, as a consequence, the topologies $\tau_{\mathrm{e}}$ and $\tau_{\mathcal{A}}$ coincide. Therefore, if $A=\mathrm{r}_{(X, \mathcal{C})}^{-1}(U)$ for some open subset $U$ of $\mathrm{r}_{\mathcal{C}} X$, it follows that $A \in \tau_{\mathcal{A}}$.

$(c) \Rightarrow(a)$ is obvious.

The following result characterizes when an epireflection functor preserves subspaces. 
Proposition 5.4. Let $\mathcal{C}$ be an epireflective subcategory of Top and let $X$ be a topological space. If $A$ is a subspace of $X$ we have that the epireflection $\mathcal{C}$ preserves the subspace $A \hookrightarrow X$ if and only if the following two properties are satisfied:

(1) For all $a_{1}, a_{2}$ in $A$ such that $r_{(A, \mathcal{C})}\left(a_{1}\right) \neq r_{(A, \mathfrak{C})}\left(a_{2}\right)$, we have $r_{(X, \mathcal{C})}\left(a_{1}\right) \neq$ $r_{(X, \mathrm{C})}\left(a_{2}\right)$;

(2) For every $\mathcal{C}$-closed (resp. $\mathcal{C}$-open) subset F of A, there is a $\mathrm{C}$-closed (resp. C-open) subset $E$ of $X$ such that $E \cap A=F$.

Proof. Suppose that $\mathcal{C}$ preserves the subspace $A \hookrightarrow X$. For simplicity, we assume that the $\mathcal{C}$-image of the subspace inclusion $A \hookrightarrow X$ is the subspace inclusion $\mathrm{r}_{\mathfrak{C}} A \hookrightarrow \mathrm{r}_{\mathfrak{C}} X$ (that is $\left.\mathrm{r}_{\mathrm{e}} A=\mathrm{r}_{(X, \mathrm{e})}(A)\right)$. Then (1) is obviously satisfied. As for (2) let $U$ be a $\mathrm{C}$-open. subset of $A$. By Lemma 5.3, there is an open subset $V$ in $\mathrm{r}_{\mathcal{C}} A$ such that $U=\mathrm{r}_{(A, \mathcal{C})}^{-1}(V)$. Furthermore, by hypothesis, we may assume that there is an open set $W$ in $\mathrm{r}_{\mathfrak{e}} X$ such that $V=\mathrm{r}_{\mathfrak{e}} A \cap W$. Thus

$$
U=\mathrm{r}_{(A, \mathcal{C})}^{-1}(V)=\mathrm{r}_{(A, \mathcal{C})}^{-1}\left(\mathrm{r}_{\mathcal{C}} A \cap W\right) \subseteq \mathrm{r}_{(X, \mathcal{C})}^{-1}\left(\mathrm{r}_{\mathcal{C}} A\right) \cap \mathrm{r}_{(A, \mathcal{C})}^{-1}(W)=\mathrm{r}_{(X, \mathcal{C})}^{-1}\left(\mathrm{r}_{\mathcal{C}} A\right) \cap \mathrm{r}_{(X, \mathcal{C})}^{-1}(W)
$$

Since also $U \subseteq A$, therefore we have

$$
U \subseteq A \cap \mathrm{r}_{(X, \mathrm{C})}^{-1}\left(\mathrm{r}_{\mathrm{e}} A\right) \cap \mathrm{r}_{(X, \mathrm{C})}^{-1}(W)
$$

and, conversely,

$$
U=\mathrm{r}_{(A, \mathcal{C})}^{-1}(V)=\mathrm{r}_{(A, \mathcal{C})}^{-1}\left(\mathrm{r}_{\mathcal{C}} A \cap W\right) \supseteq A \cap \mathrm{r}_{(X, \mathcal{C})}^{-1}(W)
$$

since $a \in A$ and $\mathrm{r}_{(X, \mathcal{C})}(a)=\mathrm{r}_{(A, \mathcal{C})}(a) \in W$ imply $\mathrm{r}_{(A, \mathcal{C})}(a) \in \mathrm{r}_{\mathcal{C}} A \cap W$. This proves that $U=\mathrm{r}_{(X, \mathrm{C})}^{-1}(W) \cap A$.

Conversely, suppose that (1) and (2) are satisfied. Let $j: A \hookrightarrow X$ be the inclusion of the subspace $A$ to $X$ and let $g: \mathrm{r}_{\mathfrak{e}} A \longrightarrow \mathrm{r}_{\mathfrak{e}} X$ be a continuous mapping that makes the following diagram 


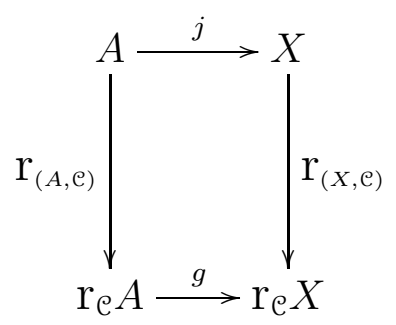

commutative

It is clear, by (1), that $g$ is injective. We will show that $g$ is also open.

Let $V$ be an open subset in $\mathrm{r}_{\mathcal{C}} A$. Then $\mathrm{r}_{(A, \mathcal{C})}^{-1}(V)$ is $\mathcal{C}$-open in $A$. By $(2)$, there is a -open subset $U$ of $X$ such that $\mathrm{r}_{(A, \mathcal{C})}^{-1}(V)=j^{-1}(U \cap j(A))$. By the commutativity of the diagram, surjectivity of $r_{(A, \mathcal{C})}$ and $r_{(X, \mathcal{C})}$, and taking into account that $U$ is the inverse image of an open set in $\mathrm{r}_{\mathfrak{C}} X$, it follows that $g(V)=\mathrm{r}_{(X, \mathcal{C})}(U) \cap \mathrm{r}_{(X, \mathcal{C})}(A)$, which is open in $\mathrm{r}_{\mathfrak{e}} A$. This completes the proof.

Definition 5.5. Let $X$ be a topological space and let $\mathcal{A}$ denote a class of topological spaces, we say that a subset $A$ of $X$ is $\mathcal{A}$-embedded when for every continuous map $f: A \rightarrow Z$, with $Z \in \mathcal{A}$, there exists $Y \in \mathcal{A}$, with $Z \subseteq Y$, a continuous map $\bar{f}: X \rightarrow Y$ such that $\bar{f}_{\mid A}=f$. In other words, every continuous map on $A$ taking values in a space in $\mathcal{A}$ can be extended to a continuous map on $X$ taking values in a possibly different larger space in $\mathcal{A}$.

Corollary 5.6. Let $X$ be a topological space and let $\mathrm{e}=\mathrm{C}(\mathcal{A})$ be the epireflective subcategory of Top that is generated by a $\mathcal{A}$. If $A$ is an $\mathcal{A}$-embedded subspace of $X$, then the epireflection functor associated to $\mathcal{C}$ preserves the subspace $A \hookrightarrow X$.

Proof. We apply Proposition 5.4 for the proof. In order to verify assertion (1), suppose that $a_{1}, a_{2}$ are two points in $A$ such that $\mathrm{r}_{(A, \mathcal{C})}\left(a_{1}\right) \neq \mathrm{r}_{(A, \mathcal{C})}\left(a_{2}\right)$. We have seen in Section 
2 that the space $\mathrm{r}_{\mathrm{e}} A$ can be realized as the diagonal of a product $\prod_{E} X=\prod_{f \in E} Y_{f}$ where $Y_{f} \in \mathcal{A}$ for all $f \in E$ and $f$ stands for a surjective continuous map $f: A \rightarrow Y_{f}$. Thus, there is a map $f: A \rightarrow Z \in \mathcal{A}$ such that $f\left(a_{1}\right) \neq f\left(a_{2}\right)$. Then there exists $Y \in \mathcal{A}$, with $Z \subseteq Y$ and a continuous map $\bar{f}: X \rightarrow Y$ that makes following diagram

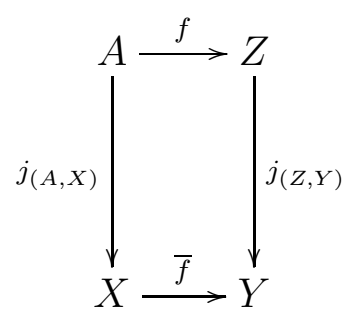

commutative. Thus, we have that $\bar{f}\left(a_{1}\right) \neq \bar{f}\left(a_{2}\right)$, which implies that $\mathrm{r}_{(X, \mathrm{e})}\left(a_{1}\right) \neq$ $\mathrm{r}_{(X, \mathrm{e})}\left(a_{2}\right)$.

As for assertion (2), it suffices to observe that the collection of $\mathcal{A}$-osets forms an open subbase for the topology $\tau_{\mathcal{A}}=\tau_{\mathfrak{e}}$.

In [25], M. Tkachenko proves that the $\mathcal{C}_{0}$-reflection respects arbitrary subgroups. The next corollary improves this result.

Corollary 5.7. The $\mathrm{C}_{0}$-reflection preserves subspaces.

Even though the $\mathcal{C}_{1}$-reflection does not preserve subspaces, our methods provide a neat characterization of this property. First we need the following lemma.

Lemma 5.8. Let $A$ be a subset of a topological space $X$. Then $A$ is $T_{1}$-closed if and only if there is a continuous mapping $f: X \rightarrow Y_{\text {cof }}$, where $Y_{\text {cof }}$ is a set equipped with the cofinite topology, such that $A=f^{-1}(p)$ for a singleton $p \in Y$. 
Proof. Suppose that $A$ is $T_{1}$-closed. Then there is a $T_{1}$-space $Z$ and a continuous mapping $g: X \longrightarrow Z$ such that $A=g^{-1}(B)$ for a closed subset $B$ in $Z$. If we identify $B$ with a singleton, say $p_{B}$, and define $Y \stackrel{\text { def }}{=}(Z \backslash B) \cup\left\{p_{B}\right\}$. Then the map $f: X \rightarrow Y_{\text {cof }}$, defined by $f(x)=g(x)$ if $x \notin g^{-1}(B)$ and $f(x)=a_{B}$ if $x \in g^{-1}(B)$, is continuous and $A=f^{-1}\left(p_{B}\right)$. The converse implication is obvious.

Since the category $\mathbf{T o p}_{1}$ of $T_{1}$-spaces is generated by the spaces equipped with the cofinite topology, we see that a subset $A$ of a topological space $X$ is $T_{1}$-embedded if and only if for every continuous map $f: A \rightarrow Z_{\text {cof }}$, where $Z$ is a set equipped with the cofinite topology, there exists a set $Y$, with $Z \subseteq Y$, a continuous map $\bar{f}: X \rightarrow Y_{\text {cof }}$ such that $\bar{f}_{\mid A}=f$.

Lemma 5.9. Every $T_{1}$-closed subspace $A$ of a topological space $X$ is $T_{1}$-embedded.

Proof. Let $f: A \rightarrow Z_{\text {cof }}^{1}$ be a continuous map defined on $A$. By Lemma 5.8, there is a continuous map $g: X \rightarrow Z_{c o f}^{2}$ and a point $p \in Z^{2}$ such that $A=g^{-1}(p)$. Set $Y \stackrel{\text { def }}{=} Z^{1} \sqcup Z^{2}$, the disjoint union of $Z^{1}$ and $Z^{2}$, and define $\tilde{f}: X \rightarrow Y_{\text {cof }}$ by $\widetilde{f}(x)=f(x)$ if $x \in A$ and $\tilde{f}(x)=g(x)$ if $x \notin A$. It is clear that inverse image by $\tilde{f}$ of every singleton in $Y$ is closed in $X$, which yields the continuity of the map.

Theorem 5.10. If $A$ is a $T_{1}$-closed subspace of a topological space $X$, then $r_{\mathrm{e}} A=$ $r_{(X, \mathrm{e})}(A)$

Proof. Apply Corollary 5.6 and Lemma 5.9.

In [25, Lemma 3.7] M. Tkachenko proved that the $T_{1}$-reflection preserves closed subgroups in the category of semitopological groups. The next Corollary is a variant of Tkachenko's result. Again, our formulation is somewhat more general. First, we 
recall that a left topological group (resp. right topological group) is a group G equipped with a topology such that the left translations $x \mapsto a x$ are continuous (resp. the right translations $x \mapsto x a$ are continuous).

Corollary 5.11. Let $G$ be a left (resp. right) topological group and let $H$ be a closed subgroup of $G$. Then $r_{\mathfrak{e}} H=r_{(G, \mathcal{C})}(H)$.

Proof. There is no loss of generality in assuming that $G$ is a left topological group. Let $X \stackrel{\text { def }}{=}(G / H)$ be the quotient space $G / H$. It is easily seen that $X$ is a $T_{1}$-space. Furthermore, if $\pi: G \rightarrow X$ denotes the canonical quotient map, we have that $H=$ $\pi^{-1}(\pi(H))$, which implies that $H$ is $T_{1}$-closed in $G$. Thus it suffices apply Theorem 5.10 .

\section{COINCIDENCE OF EPIREFLECTIONS}

The following general question is dealt with in this section: Let $\mathcal{C}$ and $\mathcal{E}$ be two epireflective subcategories of Top such that $\mathcal{\varrho} \supseteqq \mathcal{E}$. Characterize the spaces $X$ such that $\mathrm{r}_{\mathfrak{e}} X=\mathcal{E}(X)$. This topic has been studied in [6, 7] where it is left as a specific open question to characterize the spaces $X$ for which $\mathrm{r}_{\mathfrak{C}_{1}} X=\mathrm{r}_{\mathfrak{C}_{3.5}} X$, where $\mathrm{r}_{\mathfrak{C}_{1}}$ and $\mathrm{r}_{\mathfrak{C}_{3.5}}$ are the epireflection functors associated to $\mathcal{C}_{1}$ and $\mathcal{C}_{3.5}$, the subcategories of $T_{1}$-spaces and $T_{3.5}$ spaces, respectively.

Our approach is based on the notion of $\mathcal{C}$-open subset that has been introduced previously. First, we recall that in the category $\mathbf{T o p}_{0}$ the epimorphisms are not the surjections, like they are for Top. Hence also epireflective has another meaning (cf. [4]). 
Theorem 6.1. Let $\mathcal{C}$ and $\mathcal{E}$ be two epireflective subcategories of Top such that Top $_{0} \supseteq$ $\mathcal{\varrho} \mathcal{E}$ and let $X$ be a topological space. Then $r_{\mathcal{C}} X=r_{\mathcal{E}} X$ if and only if every $\mathcal{C}$-open subset of $X$ is $\mathcal{E}$-open.

Proof. The "only if" part is obvious. As for the "if" part, consider the following commutative diagram

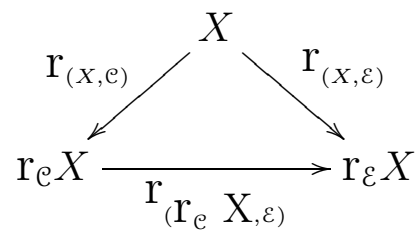

where $r_{\left(r_{e} X, \mathcal{E}\right)}$ is the unique continuous map canonically defined since $\mathcal{C} \supseteqq \mathcal{E}$. It will suffice to verify that $\mathrm{r}_{\left(\mathrm{r}_{\mathrm{e} X, \varepsilon)}\right.}$ is 1-to-1 and open. Suppose first that $x, y$ belong to $X$ and $\mathrm{r}_{(X, \mathcal{e})}(x) \neq \mathrm{r}_{(X, \mathcal{e})}(y)$. By our initial assumption $\mathcal{C}$ is included in $\mathbf{T o p}_{0}$. Thus there is an open subset $W$ in $\mathrm{r}_{\mathrm{e}} X$ that contains exactly one of these points. Assume wlog that $\mathrm{r}_{(X, \mathcal{e})}(x) \in W \not \ngtr \mathrm{r}_{(X, \mathrm{e})}(y)$, which yields $x \in \mathrm{r}_{(X, \mathrm{e})}^{-1}(W) \not \supset y$. By hypothesis and Lemma 5.3, there must be an open subset $V$ of $\mathrm{r}_{\mathcal{E}} X$ such that $\mathrm{r}_{(X, \mathcal{C})}^{-1}(W)=\mathrm{r}_{(X, \mathcal{E})}^{-1}(V)$.

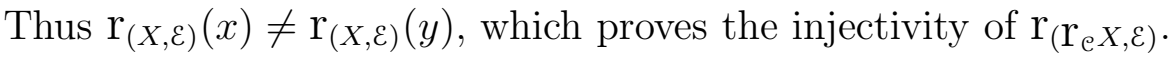

Now, let $W$ be an arbitrary open subset of $\mathrm{r}_{\mathfrak{e}} X$. Again, there must be an open subset $V$ of $\mathrm{r}_{\mathcal{E}} X$ such that $\mathrm{r}_{(X, \mathcal{C})}^{-1}(W)=\mathrm{r}_{(X, \mathcal{E})}^{-1}(V)$. Furthermore, the commutativity of the diagram above implies that $\mathrm{r}_{(X, \mathrm{e})}^{-1}(W)=\mathrm{r}_{(X, \mathcal{C})}^{-1}\left(\mathrm{r}_{\left(\mathrm{r}_{\mathrm{e} X, \mathcal{E})}^{-1}\right.}^{-1}(V)\right)$. This implies that

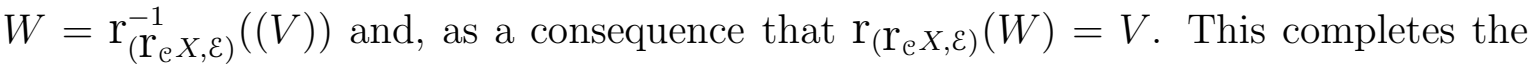
proof.

The following result answers Question 1.6 in [6], repeated in [7, Question 1.9]. 
Corollary 6.2. Let $X$ be a topological space. Then $r_{\mathfrak{C}_{1}} X=r_{\mathcal{C}_{3.5}} X$ if and only if every $T_{1}$-closed subset $F$ of $X$ is completely separated from any point $x \notin F$.

Proof. Necessity: Suppose that $\mathrm{r}_{\mathfrak{C}_{1}} X=\mathrm{r}_{\mathfrak{C}_{3.5}} X$. By Theorem 6.1, every $T_{1}$-closed subset $F$ of $X$ is $\mathcal{C}_{3.5}$-closed. Therefore, by Lemma $\left[5.3\right.$, there is a closed subset $E \subseteq \mathrm{r}_{\mathfrak{C}_{3.5}} X$ such that $F=\mathrm{r}_{(X, \mathcal{C})}^{-1}(E)$. Thus, $\mathrm{r}_{(X, \mathcal{C})}(x) \notin E$ for all $x \notin F$. Since $\mathrm{r}_{\mathcal{C}_{3.5}} X$ a $T_{3.5}$ space, this implies that $F$ is completely separated from any point $x \notin F$.

Sufficiency: Let $U$ be a $T_{1}$-open subset of $X$, we must verify that $U$ is $\mathcal{C}_{3.5}$-open in order to apply Theorem 6.1. By hypothesis $X \backslash U$ is completely separated from any point $x \in U$. Hence $X \backslash U=\mathrm{r}_{\left(X, \mathrm{e}_{3.5}\right)}^{-1}\left(\mathrm{r}_{\left(X, \mathrm{e}_{3.5}\right)}(X \backslash U)\right)$, which implies that $X \backslash U$ is $\mathcal{C}_{3.5}$-closed and therefore $U$ must be a $\mathcal{C}_{3.5}$-open subset of $X$, which completes the proof.

\section{REFERENCES}

[1] J. Adámek, H. Herrlich, G. E. Strecker, Abstract and Concrete Categories, The Joy of Cats, Wiley, New York etc., 1990.

[2] P. Alexandroff, On the concept of a topological space, Uspehi Mat. Nauk (NS) 2, no 1 (17), (1947), $5-57$.

[3] A. Arhangelskii, M. Tkachenko, Topological Groups and related structures, Atlantis Studies in Mathematics, 2008.

[4] S. Baron, Note on epi in $\tau_{0}$, Canad. Math. Bull. 11, (1968), 503-504.

[5] P. M. Cohn, Universal algebra, Reidel, 1981.

[6] O. Echi and S. Lazaar, Universal spaces, Tychonoff and spectral spaces, Math. Proc. R. Ir. Acad., 109A, (1), (2009), 35-48.

[7] O. Echi and S. Lazaar, Reflective subcategories, Tychonoff spaces, and spectral spaces., Topol. Proc., 34 (2009), 307-319.

[8] P.M. Gartside, E.A. Reznichenko, and O.V. Sipacheva, Mal'tsev and retral spaces, Top. Appl., 80 (1997), 115-129.

[9] E. Giuli, Bases of topological epi-reflections, Top. Appl., 11 (1980) 265-273.

[10] G. Grätzer, Universal algebra, Van Nostrand, Princeton, N.J. - Toronto, Ont. - London, 1968.

[11] J. E. Hart and K. Kunen, Bohr compactifications of discrete structures, Fundam. Math., 160, (2) (1999), 101-151.

[12] H. Herrlich, On the concept of reflections in general topology., Contrib. Extens. Theory Topol. Struct., Proc. Sympos. Berlin 1967 (1969) 105-114. 
[13] H. Herrlich, G. Strecker, Categorical topology - its origins, as exemplified by the unfolding of the theory of topological reflections and coreflections before 1971, Handbook of the History of General Topology, Vol. 1, 255-341, Kluwer, Dordrecht, 1997.

[14] H. Herrlich, G. E. Strecker, Category Theory: an Introduction, 3rd ed., Sigma Series in Pure Math., 1, Heldermann, Lemgo, 2007.

[15] M. Hušek and J. de Vries, Preservation of products by functors close to reflectors., Topology Appl., 27 (1987), 171-189.

[16] J. F. Kennison, Reflective functors in general topology and elsewhere, Trans. Amer. Math. Soc., 118 (1965), 303-315.

[17] A. G. Kurosh, Lectures on general algebra, Chelsea, 1963 (translated from Russian).

[18] F. Lin, K. Zhang, A problem of M. Tkachenko on semitopological groups, Top. Appl., 172 (2014), $10-13$.

[19] A. I. Mal'tsev, On the general theory of algebraic systems, Mat. Sb. (N.S.), 35 (77) (1954), 3-20.

[20] K. Morita, J. Nagata, Topics in General Topology, Vol. 41. North-Holland Mathematical Library. 1989.

[21] Liang-Xue Peng, A note on semitopological groups and paratopological groups, Top. Appl., 191 (2015) 143-152.

[22] O.V. Ravsky, Paratopological Groups II, Mat. Stud., 17 (2002), 93-101.

[23] E.A. Reznichenko and V.V. Uspenskij, Pseudocompact Mal'tsev spaces, Top. Appl., 86, (1) (1998), 83-104.

[24] I. Sánchez, A note on the $T_{2}$-reflection of semitopological groups, Top. Appl., 182 (2015) 117-121.

[25] M. Tkachenko, Axioms of separation in paratopological groups and related functors, Top. Appl., 179 (2014), 200-214.

[26] M. Tkachenko, Axioms of separation in semitopological groups and related functors, Top. Appl., 161 (2014), 364-376.

[27] M. Tkachenko, Applications of the reflection functors in paratopological groups, Top. Appl., 192 (2015), 176-187.

[28] V. V. Uspenskij, The topological group generated by a Lindelöf $\Sigma$-space has the Souslin property, Soviet Math. Dokl., 26 (1982), 166-169.

[29] A. N. Whitehead, A treatise on universal algebra, with applications, Cambridge Univ. Press, 1898.

[30] Li-Hong Xiea, Piyu Li, Jin-Ji Tuc, Notes on (regular) $T_{3}$-reflections in the category of semitopological groups, Top. APPL., 178 (2014), 46-55.

Universidad de Cartagena, Departamento de Matemáticas, Campus de San Pablo, Cartagena, Colombia.

E-mail address: jhernandeza2@unicartagena.edu.co

Universitat Jaume I, Departamento de Matemáticas, Campus de Riu Sec, 12071 Castellón, SPAIN.

E-mail address: hernande@uji.es 